\title{
LES FORÊTS EN OUTRE-MER : UN ENJEU MONDIAL POUR LA BIODIVERSITÉ
}

\section{Jacques Trouvilliez - Frédéric Mortier}

\section{DES FORÊTS VARIÉES}

2011, l'année des Outre-mer pour le gouvernement français, met en valeur la biodiversité exceptionnelle des territoires ultramarins, tant au niveau des espèces qu'au niveau des écosystèmes. Il est couramment estimé que $80 \%$ de la biodiversité nationale se trouvent outre-mer. De plus, $98 \%$ de la flore supérieure et $96 \%$ de la faune vertébrée endémiques françaises sont ultramarines. Selon le bilan de la liste rouge mondiale publiée par l'UICN, la France figure avec ces territoires parmi les dix pays hébergeant le plus grand nombre d'espèces mondialement menacées.

Douze territoires (figure 1, ci-dessous) constituent l'outre-mer français. Ils ont un statut administratif varié : certains sont des départements d'outre-mer (DOM) où l'État exerce les mêmes compétences qu'en métropole alors que d'autres sont des collectivités ayant pleinement compétence sur l'environnement. Les milieux forestiers sont à l'image de ces territoires, leur variété est grande depuis la forêt amazonienne du massif guyanais jusqu'aux forêts boréales de Saint-Pierreet-Miquelon, sans omettre les forêts de montagne de l'île de la Réunion ou les mangroves des littoraux tropicaux. Le taux de boisement des départements d'outre-mer est supérieur à celui des départements métropolitains mais la Guadeloupe, la Martinique et la Réunion ont un taux d'arti-

\section{FIGURE 1 LOCALISATION DES 12 COLLECTIVITÉS}

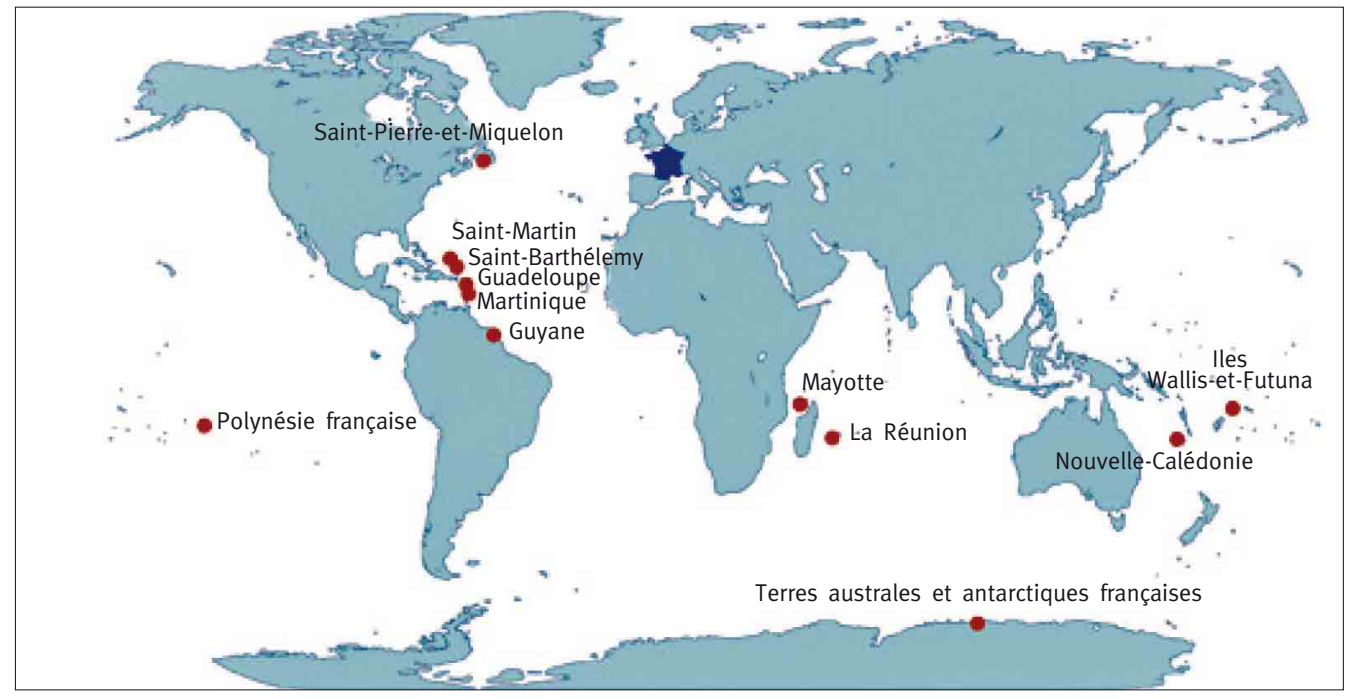



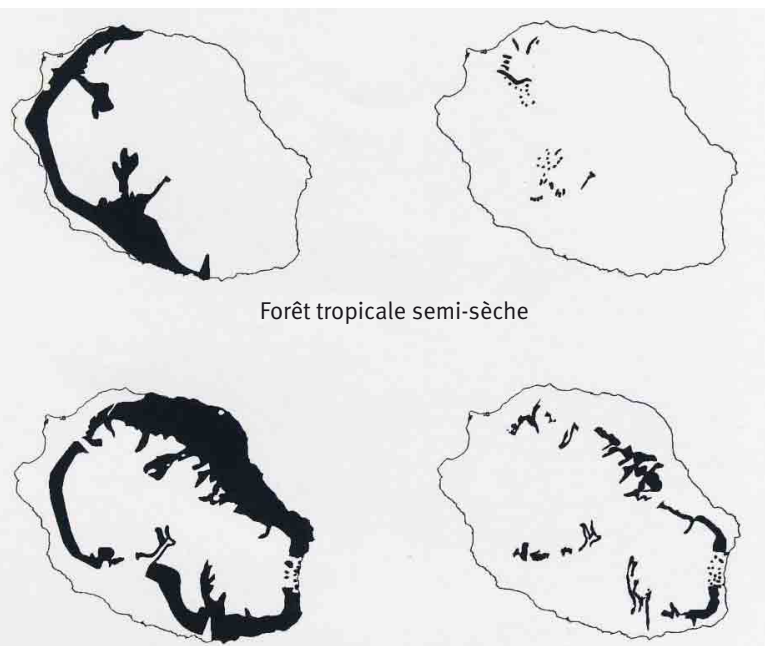

Forêt tropicale humide
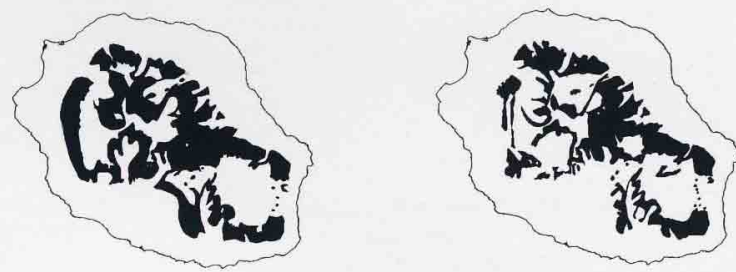

Forêt de montagne

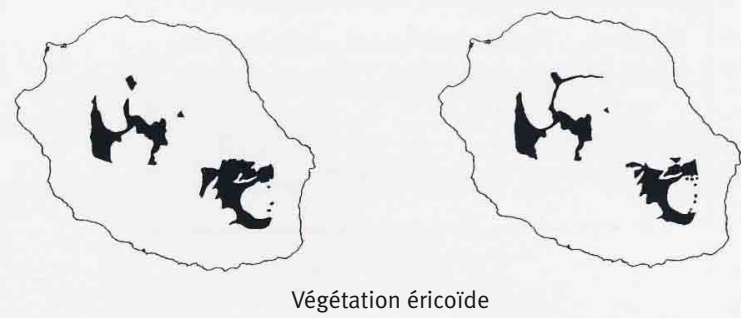

FIGURE 2 ÉVOLUTION

DES GRANDS TYPES DE VÉGÉTATION

SUR L'ILE DE LA RÉUNION

ENTRE 1665 ET AUJOURD'HUI

Sources : T. Cadet complété par

J. Dupont, SREPEN, Diren

et Parc national de la Réunion

in Robert (R.). - île de la Réunion, un patrimoine d'exceptions. - Parc national de la Réunion éd., 2009

ficialisation des sols supérieur. Le statut foncier des forêts est lui-même particulier : privé, communautaire, départementalo-domanial (pour trois DOM) ou domanial.

Ces territoires connaissent aussi des situations démographiques contrastées mais toutes posent la question de la conservation des forêts dans des termes peu connus en métropole, où la stabilité voire l'extension du manteau forestier est la règle. La pression foncière, les occupations illégales, certaines exploitations minières constituent souvent le quotidien des gestionnaires.

Dans le Pacifique, en Nouvelle-Calédonie, $22 \%$ du territoire terrestre est forestier (environ $4400 \mathrm{~km}^{2}$ ), avec deux grands types majeurs : forêts humides sur la côte est et forêts sèches sur la côte ouest. À Wallis-etFutuna, $10 \%$ de la superficie des îles est forestière $\left(27,4 \mathrm{~km}^{2}\right)$. En Polynésie où l'on compte 120 îles hautes volcaniques et basses coralliennes,

la forêt couvre $40 \%$ des terres, mais les forêts naturelles seulement 14 \%.

Dans l'océan Indien, la couverture forestière est relativement élevée : 36 \% à la Réunion et $42 \%$ à Mayotte, mais beaucoup de forêts naturelles ont disparu, notamment à basse altitude où les forêts sèches de la Réunion ne représentent plus que $3 \%$ des superficies originelles (figure 2 , ci-dessus).

Dans les Caraïbes, la couverture forestière est encore forte : $48 \%$ en Guadeloupe $\left(823 \mathrm{~km}^{2}\right)$ et $41 \%$ en Martinique $\left(465 \mathrm{~km}^{2}\right)$, mais en dessous de $600 \mathrm{~m}$ les forêts sont très fragmentées notamment sur Grande-Terre en Guadeloupe.

Le massif forestier guyanais, le plus important de France (80 $\left.492 \mathrm{~km}^{2}\right)$, appartient à l'un des trois grands bassins forestiers tropicaux. 
Enfin les forêts boréales de Saint-Pierre-et-Miquelon, de superficie réduite $\left(60 \mathrm{~km}^{2}, 24,8 \%\right.$ de l'archipel), complètent la palette des milieux forestiers.

\section{UN TAUX D’ENDÉMICITÉ TRÈS VARIABLE}

Les forêts d'outre-mer se rencontrent sur des continents (Guyane), sur des îles isolées d'un continent (parfois depuis très longtemps, cas de la Nouvelle-Calédonie, détachée du Gondwana, continent ancestral), ou sur des îles océaniques nées du fond des océans à la suite d'événements volcaniques (la Réunion par exemple). L'histoire géologique de ces territoires, leur âge et leur relatif isolement par rapport aux terres voisines expliquent en grande partie la composition de leur flore et des forêts. La biodiversité terrestre présente en général un taux d'endémicité supérieur à celui de la biodiversité marine, les courants dispersant efficacement les espèces.

Ce sont ainsi les îles les plus anciennes et les plus isolées qui présentent le taux d'endémisme le plus élevé. La Nouvelle-Calédonie détient ainsi la "palme » au sein de l'outre-mer : 74 \% pour les plantes vasculaires (3 261 espèces) et $66 \%$ pour les chiroptères. Il s'agit d'un des taux d'endémisme les plus élevés de la planète. Les Gymnospermes sont représentées par 44 espèces, dont 43 conifères tous endémiques, soit $7 \%$ des espèces de la planète (par exemple Parasitaxus ustus, seul conifère parasite au monde et 19 espèces d'Araucaria).

En Polynésie, ce taux est estimé à 60 \% pour les plantes vasculaires (850 espèces).

Dans l'océan Indien, Mayotte et trois des îles Éparses sont proches du continent africain et de Madagascar et leur flore y est apparentée, le taux d'endémicité est faible, comme attendu depuis les travaux de MacArthur et Wilson sur la biogéographie des îles publiés en 1967. La Réunion est relativement isolée mais sa " jeunesse " géologique jouant sur la colonisation et les processus de spéciation explique un taux d'endémicité " moyen » : $34 \%$ des plantes à fleurs.

En Guyane, les connaissances sont encore fragmentaires, mais l'endémicité est évaluée à 3,5\% pour les plantes supérieures (5 120 espèces) ; ceci s'explique par l'appartenance de la Guyane $\mathrm{au}$ vaste ensemble forestier de l'Amazonie, même si les inselbergs ont favorisé l'isolement de certains peuplements végétaux et leur singularité.

\section{DES TERRITOIRES RICHES ET MENACÉS}

Myers et al. (2000) ont, les premiers, identifié 25 "hotspots de la biodiversité» sur la base de deux critères qui doivent être tous deux remplis pour qu'un ensemble biogéographique puisse être ainsi qualifié : une concentration d'espèces endémiques très élevée et un taux de disparition des habitats naturels élevés. Ce travail visait à fixer des priorités de conservation là où les territoires sont riches et où les menaces exigent des actions urgentes pour éviter la disparition de cette richesse unique au monde. Ces 25 points chauds comprennent $44 \%$ des plantes vasculaires connues sur $1,4 \%$ de la superficie terrestre de la planète.

Parmi ces 25 hotspots mondiaux, quatre concernent l'outre-mer français :

- la Nouvelle-Calédonie en tant que telle, troisième en termes de densité d'espèces végétales endémiques,

- la Polynésie française au sein du plus vaste hotspot Polynésie-Micronésie,

- l'île de la Réunion, Mayotte et les îles Éparses au sein d'un ensemble regroupant Madagascar, les Comores, les Seychelles, Maurice et Rodrigues, 
- la Guadeloupe, la Martinique, Saint-Martin et Saint-Barthélemy au sein du hotspot Caraïbes qui s'étend depuis Trinidad jusqu'au sud de la Floride.

La Guyane et le bassin amazonien ne figurent pas parmi ces points chauds, ne remplissant pas les deux critères ci-dessus (concentration d'endémiques et menace de disparition des habitats naturels) mais cela ne signifie pas que ce patrimoine n'est pas remarquable.

À la suite de ces travaux, le partenariat Critical Ecosystem Partnership Fund, associant notamment l'ONG Conservation International, la Banque mondiale et l'Agence française pour le développement, consacre prioritairement des moyens à la sauvegarde de ces milieux et des espèces qui s'y rencontrent.

Cette approche des points chauds souligne la responsabilité mondiale française en matière de conservation de la nature.

\section{LES MENACES}

Au niveau mondial, les principales menaces qui pèsent sur la biodiversité sont la destruction, l'altération et la fragmentation des habitats naturels, d'une part, et d'autre part les espèces exotiques envahissantes, auxquelles se surimpose le changement climatique.

Au cours de la colonisation des différents territoires, les forêts ont fortement régressé, phénomène classique connu par ailleurs : les populations chassent, défrichent pour élever le bétail et mettre en culture, exploitent le bois comme matériau et combustible, parfois en bouleversant profondément le fonctionnement des écosystèmes (Diamond, 2005). Dans les îles, ce sont les forêts sèches qui paient le plus lourd tribut : moins de $3 \%$ subsistent à la Réunion et moins de $1 \%$ en Nouvelle-Calédonie. À ces suppressions de couvert forestier, s'ajoute l'altération des forêts naturelles. Ainsi sur les îles basses coralliennes de Polynésie (forêts à Puatea et Pandanus), elles ont été remplacées par des cocoteraies. Les forêts humides, notamment lorsqu'elles sont en altitude sont mieux conservées. Toutefois à Wallis-et-Futuna, par exemple, il ne reste plus que $10 \%$ de la forêt dense humide ( $3000 \mathrm{ha}$ ) suite aux différentes vagues de colonisation. L'étude récente (juin 2011) du Service de l'observation et des statistiques (SOeS, CGDD, MEDDTL) montre que la destruction des habitats naturels dans les DOM est relativement faible en superficie, mais que les besoins en infrastructures et en urbanisme consomment les espaces naturels et notamment forestiers, notamment près des côtes. Dans la bande littorale des $20 \mathrm{~km}$ de Guyane, 20000 ha ont été affectés par des changements d'occupation des sols entre 2000 et 2006 . L'essor démographique de ces territoires et de leurs voisins font peser des menaces sur le devenir des forêts encore perçues comme réserves foncières en bien des endroits. En Guyane, le terme d'explosion démographique serait plus approprié avec un taux de natalité de 31 pour mille et une immigration, y compris illégale, massive. La population qui était de 23000 habitants en 1946 atteint 215000 en 2007, et les projections démographiques envisagent une fourchette basse de 425000 habitants à l'horizon, proche !, de 2030 (fourchette haute de 600000 habitants). La culture sur abattis, parfois liée à des défrichements illégaux, est un moyen de survie et d'insertion sociale (Tsayem Demaze, 2008). Toutefois, la superficie des mangroves de Guyane ne semble pas dépendre de la pression urbaine, mais des courants marins et des phénomènes de sédimentation. Cette forêt particulière d'interface se trouve outre-mer aux deux tiers en Guyane, pour $25 \%$ en Nouvelle-Calédonie puis pour 6 \% dans les Caraïbes (Roussel, 2009).

Les espèces exotiques envahissantes sont une menace que certains ont qualifiée d'invisible mais bien réelle. Les écosystèmes insulaires sont plus fragiles que les écosystèmes continentaux pour plusieurs raisons : cortège floristique moins riche, compétition interspécifique plus faible et désé- 
quilibre fonctionnel, certains groupes biologiques étant absents (Williamson, 1997). Les exemples sont nombreux dans tout l'outre-mer (Soubeyran, 2008 ; comité français de l'UICN et ONCFS, 2011). Parmi la liste établie par l'UICN au niveau international des 100 espèces figurant parmi les plus envahissantes du monde, 49 sont déjà présentes dans l'outre-mer français (Acacia mearnsii, Cecropia peltata ou Melaleuca quinquenervia pour ne citer que quelques arbres). Le cas de Miconia calvescens dans les îles de la Société représente l'un des cas les plus spectaculaires d'invasion biologique par une plante introduite dans un écosystème insulaire tropical. Petit arbre de 6 à $8 \mathrm{~m}$ de hauteur, originaire d'Amérique centrale et du Sud, il est introduit comme plante ornementale en 1937 à Tahiti dans un jardin botanique. Comme souvent, son caractère envahissant n'est remarqué que dans les années 1970, et désormais les deux tiers de l'île de Tahiti sont envahis, Miconia formant des couverts denses monospécifiques menaçant directement 70 espèces de plantes locales. Le Troène de Ceylan, Ligustrum walkeri, introduit volontairement à la Réunion bouleverse la composition floristique des forêts humides, ses fruits étant notamment dispersés par une autre espèce invasive, le Bulbul orphée, Pycnonotus jocosus. L'introduction d'espèces animales pèse aussi sur le devenir des écosystèmes forestiers. À Saint-Pierre-et-Miquelon, l'explosion démographique du cerf de Virginie (Odocoileus virginianus) et du lièvre d’Amérique (Lepus americanus), espèces introduites (en 1953 pour le cerf), empêche désormais toute régénération de la forêt.

Les activités humaines comme l'orpaillage en Guyane et l'exploitation minière en Nouvelle-Calédonie (nickel, cobalt et chrome) bouleversent les cours d'eau et les sols et modifient le fonctionnement de l'écosystème (hydrologie, pollution, défrichement...).

Enfin, le changement climatique pèse aussi sur le devenir des forêts (cf. l'article de Roman-Amat et al. dans ce numéro), y compris outre-mer. Ponteau et al. (2010) ont simulé en 2050 et 2100 le devenir de la forêt de nuages (forêt ombrophile d'altitude) qui perdrait en un siècle $90 \%$ de sa superficie et se fragmenterait. De nombreuses espèces endémiques verraient alors leur état de conservation très fortement dégradé.

\section{LES ÉVOLUTIONS dANS LA CONNAISSANCE ET LES POLITIQUES PUBLIQUES}

La prise en compte des richesses patrimoniales de l'outre-mer est relativement récente.

L'exemple des forêts sèches de Nouvelle-Calédonie traduit bien cette lente reconnaissance. Ces forêts sont parmi les plus riches de la planète, mais pendant longtemps elles ont été négligées. En forte régression ( $1 \%$ de la superficie originelle !), ces forêts sont morcelées (la plupart des lambeaux existants font moins de $5 \mathrm{ha}$ ) et ne totalisent plus que $45 \mathrm{~km}^{2}$ (et même 1932 ha pour les forêts sclérophylles en bon état de conservation !). On dénombre 59 espèces de plantes qui sont des endémiques strictes de ces forêts. Certaines espèces à fort potentiel agronomique comme le riz adapté à la sécheresse Oryza neocaledonica sont inféodées à ces milieux. En 1956, l'inspecteur des forêts Maurice Virot plaçait ces forêts dans les formations littorales et, en 1981, les botanistes individualisaient ces formations que l'ORSTOM cartographia en 1990 dans la province Sud. En 1994, une première parcelle fut mise en défens et, en 1995, des scientifiques (Bouchet et al.) insistaient sur l'urgence à intervenir. Le WWF propose alors un programme de sauvegarde et, en 2001, 9 partenaires s'engagent sur un programme global de conservation qui cible des sites prioritaires. Ces partenaires sont institutionnels (État français, gouvernement de Nouvelle-Calédonie, provinces Nord et Sud), scientifiques (institut agronomique néocalédonien, IRD et université de Nouvelle-Calédonie) et associatifs (WWF-France et centre d'initiation à l'environnement). Afin notamment de pérenniser cette action exemplaire, un groupement d'intérêt public, le Conservatoire des espaces naturels de Nouvelle-Calédonie, vient d'être créé en 2011, associant tous les partenaires publics concernés. 
D’autres conservatoires des espaces naturels se mettent en place à la Réunion et en Guyane.

L'Office national des forêts, présent dans les DOM, assure une protection foncière mais aussi des actions de conservation notamment par une politique active de création de réserves biologiques et par la lutte contre l'envahissement des milieux par les espèces exotiques.

\section{LE MOUVEMENT DE CONSERVATION S'ACCÉLÈRE}

Après la création en 1989 du premier parc national ultramarin en Guadeloupe, deux nouveaux parcs nationaux ont été créés en 2007 en Guyane (encadré 2, page 547) et à la Réunion, après la promulgation de la Loi sur les parcs nationaux du 14 avril 2006. De nombreuses réserves naturelles ou espaces protégés ont vu le jour sur l'ensemble de l'outre-mer, le cas de Wallis-et-Futuna étant une exception. L'UICN (2010) a d'ailleurs souligné cet accroissement significatif mais déplore que le projet de stratégie de création des aires protégées ne s'applique qu'à la métropole.

À la suite du Grenelle de l'environnement, l'engagement de rédiger des plans d'action pour les espèces en danger critique d'extinction révèle la richesse de l'outre-mer français (sur 131 espèces concernant la France, 35 espèces concernent la Nouvelle-Calédonie et 54 espèces la Polynésie). Ce recensement extrait de la liste rouge mondiale 2007 de l'UICN a également révélé que l'outremer français est peu connu au sein des instances internationales : des erreurs ont été notées et la liste a pu surprendre. Depuis, le comité français de l'UICN a relancé le travail sur les listes rouges et les premières listes actualisées pour l'outre-mer sont parues. La liste concernant la Réunion en 2010 révèle que $30 \%$ des espèces de la flore sont menacées.

Le comité français de l'UICN cherche à sensibiliser l'opinion sur la biodiversité ultramarine française (un groupe de travail spécifique a été créé) et plusieurs documents de synthèse ont été publiés pour sensibiliser tous les acteurs : espèces exotiques envahissantes (Soubeyran, 2008), appui à la stratégie nationale pour la biodiversité (SNB) initiée par l'État en 2004 et au plan d'action outre-mer, apports lors de la révision de la SNB en 2011. Au niveau européen, l'UICN a fait la revue de la mise en œuvre de la convention sur la diversité biologique et des plans d'action dans l'outre-mer européen (2010) en vue de la conférence de Nagoya.

En juillet 2008, dans le cadre de la présidence française du Conseil de l'Union européenne, à l'initiative du conseil régional de la Réunion et de l'ONERC, s'est tenue une conférence " l'Union européenne et l'Outre-mer : Stratégies face au changement climatique et à la perte de biodiversité ». Le message issu de cette conférence a été repris le 25 juin 2009 par le Conseil européen et, en mai 2011, une action préparatoire dotée de deux millions d'euros a lancé le programme BEST (programme volontaire pour la biodiversité, les écosystèmes et les services écosystémiques dans les territoires d'outre-mer européens). Ce programme vise à promouvoir la conservation et l'utilisation durable de la biodiversité et des services écosystémiques en outre-mer en s'inspirant des expériences menées dans l'Union européenne en matière de conservation de la nature. Les projets pilotes seront lancés en 2012.

La politique de la connaissance qui se structure en métropole au travers du système d'information sur la nature et les paysages (SINP) piloté par le ministère chargé de l'Environnement conduit parallèlement à relancer l'acquisition des connaissances en outre-mer. En effet si la flore de Nouvelle-Calédonie est l'une des flores tropicales les mieux inventoriées au monde, de vastes zones restent encore à prospecter et la biologie de certaines espèces menacées n'est pas connue, ce qui rend plus difficile les actions de sauvegarde. L'ouvrage de référence de Bernard Rollet sur les arbres des Petites Antilles est enfin paru en 2010 tout comme celui de Pierre Charles-Dominique sur les milieux, la faune et la flore de Guyane en 2011. La Réunion a été le premier dépar- 
tement d'outre-mer à bénéficier d'un conservatoire botanique agréé. Mais les autres initiatives similaires tardent à aboutir ou sont embryonnaires, alors que ces outils de connaissance sont un modèle qu'il serait opportun de développer pour mieux asseoir les stratégies de conservation. Un projet de création existe en Guyane.

L'initiative française pour la conservation et la gestion de la biodiversité outre-mer (IFREBIOM), souhaitée par les états généraux de l'outre-mer et validée par le conseil interministériel de l'outre-mer le 6 novembre 2009, doit être l'occasion de mieux faire connaître et échanger les expériences de conservation, à l'instar de l'IFRECOR pour les récifs coralliens. Cette initiative pourrait être lancée en 2012.

Le cadre réglementaire en place sur l'introduction des espèces exotiques susceptibles d'être envahissantes ne permet pas une gestion appropriée du risque que représente cette menace particulièrement importante dans les écosystèmes insulaires qui sont les plus vulnérables. Mais chaque territoire a élaboré une stratégie contre ces espèces, utilisant, faute de mieux, les régimes zoo- et phytosanitaires notamment et de nombreux programmes de sensibilisation et de lutte se mettent en place. Le comité français de l'UICN coordonne une initiative impliquant tous les territoires et un très large éventail d'acteurs, mutualisant les techniques de lutte. Il faut en effet prendre garde à restaurer le milieu et éviter que, par effet de cascade, une espèce invasive ne vienne prendre la place d'une autre espèce invasive récemment éradiquée comme l'ont montré les travaux entrepris par l'ONF à la Réunion.

En Polynésie, sur l'île de Raïatea, des campagnes de lutte annuelles contre Miconia sont menées depuis 1992, stoppant l'invasion sans toutefois éradiquer définitivement l'espèce du fait de la longévité des graines dans le sol et de la difficulté d'accès de certaines zones. La lutte biologique a démarré en 2000 sur Tahiti et 2004 sur Raïatea, utilisant un champignon pathogène (Colletotricum gloeosporioides), mais si tous les pieds de Miconia sont contaminés, le taux de mortalité est faible et n'a pas produit les résultats espérés.

\section{CONCLUSIONS}

La France a ratifié la convention sur la diversité biologique en 1994 ; cette convention s'applique désormais à tous les territoires français d'outre-mer, ce qui n'est pas le cas pour d'autres outremer européens. En application de cette convention, un plan d'action spécifique pour l'outre-mer (et des déclinaisons par territoire) a été adopté en 2006, mais son application a été inégale. En octobre 2010, à Nagoya, un plan stratégique 2011-2020 a été adopté. Parmi les 20 objectifs, le rythme d'appauvrissement de tous les habitats naturels, y compris les forêts, doit être réduit de moitié et si possible tendre vers zéro. La stratégie nationale pour la biodiversité a été lancée le 19 juin 2011 par la ministre chargée de l'Environnement. Cette nouvelle stratégie, davantage fondée sur l'implication des acteurs, devrait permettre un engagement plus important des collectivités, et notamment de celles d'outre-mer qui portent la majeure partie de la biodiversité nationale.

La conservation de la biodiversité dans l'outre-mer français, et plus particulièrement la biodiversité forestière, pose avec une acuité propre des questions déjà difficiles à traiter en métropole. Comment articuler les aspirations légitimes au développement avec la conservation de ces milieux exceptionnels ? La stabilisation des situations foncières et des autres pressions n'est pas atteinte, occupations et exploitations illégales sont des facteurs localement importants. Les défis à relever dans ces territoires sont comparables à ceux qui se rencontrent dans d'autres hotspots mondiaux de la biodiversité. L'évolution de la gouvernance pour davantage de concertation entre les propriétaires, quels qu'ils soient, les acteurs politiques et les ONG se pose avec acuité si nous voulons favoriser la conservation. Le développement endogène des territoires ultramarins passe 
aussi par la biodiversité, sa valorisation dans le domaine du tourisme et la filière bois élargie aux ressources biologiques. Il est nécessaire de diversifier les moyens financiers mobilisables en faveur de la biodiversité, des biens et services rendus par la forêt au-delà du seul bois. La nouvelle stratégie pour la biodiversité donne un cadre partenarial pour écrire ensemble l'avenir de ces territoires et de leur exceptionnelle richesse.

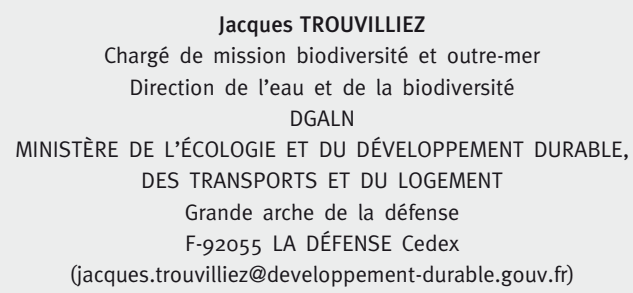

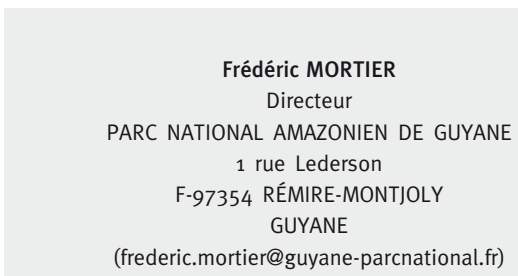

\section{Remerciements}

à : Nathalie Bertin (PAG), Guillaume Feuillet (PAG), Michel Hermeline (DG ONF), Patrice Hirbec (ONF/DG), Marylène Hoarau (PN Réunion), Hervé Houin (ONF Réunion), Pierre Joubert (PAG), Nicolas Karr (ONF Guyane), Hervé Magnin (PN Guadeloupe), Rosiane Mandé (PAG), Patrice Mengin-Lecreulx (ONF/DG), Jean-Maurice Montoute (PAG), Denis Nebel (ONF Martinique), Claude Rupé (DG ONF), Mylène Valentin (ONF Guadeloupe), Jacques Valeix (IG ONF).

\section{1 - Les réserves biologiques : un outil de conservation dans les forêts publiques}

Au sein des forêts publiques peuvent être créées des réserves biologiques, forestières ou domaniales selon le statut de propriété, intégrales (RBI) ou dirigées (RBD) selon les objectifs poursuivis (conservation des processus en libre évolution ou bien gestion orientée vers la conservation d'éléments particuliers du patrimoine).

Les réserves biologiques actuelles, faisant l'objet d'un arrêté interministériel ou ayant reçu un avis favorable du Conseil national de la protection de la nature, représentent 97500 ha dans les DOM, dont 89200 ha dans sept réserves intégrales et 8300 ha dans sept réserves dirigées. Les forêts domaniales ou départementalo-domaniales y sont très majoritaires.

La Guyane possède la plus grande RBI (60 960 ha : Lucifer Dékou-Dékou). La Réunion compte 25150 ha en RBI et 8320 ha en RBD. Enfin, 3073 ha sont classés en RBI à la Martinique.

Ces réserves biologiques couvrent essentiellement des milieux de forêts tropicales humides.

Plusieurs projets sont en cours, notamment en Guadeloupe avec une RBI de plusieurs centaines d'hectares qui englobera des forêts sèches à Grande-Terre. À la Martinique, une RBI de 3300 ha est en cours d'instruction (Pitons du Carbet), ainsi que plusieurs RBD recouvrant des enjeux spécifiques : sites de ponte de tortues marines, site de ponte de sternes de Dougall, ou plantations de Mahogany en voie de renaturalisation.

En outre, six réserves naturelles nationales concernent également des terrains gérés par l'ONF dans les DOM, pour une surface de 183600 ha. 


\section{2 - Le parc amazonien de Guyane : un géant aux défis d'exception}

Le parc amazonien de Guyane, créé le 27 février 2007, est le parc national le plus vaste de France et de l'Union européenne (3,4 millions d'hectares dans la moitié sud de la Guyane française ; figure 3, ci-dessous). Le cœur du parc, zone dédiée à la conservation de la nature, s'étend sur 2 millions d'hectares. La zone d'adhésion de 1,4 million d'hectares est un espace qui associe protection de l'environnement et développement économique, local et durable, avec et pour les populations. Ce parc national est frontalier du parc national des monts Tumucumaque (3,9 millions d'hectares) situé dans l'État d'Amapa au Brésil ; l'ensemble constitue le plus grand espace forestier protégé au monde.

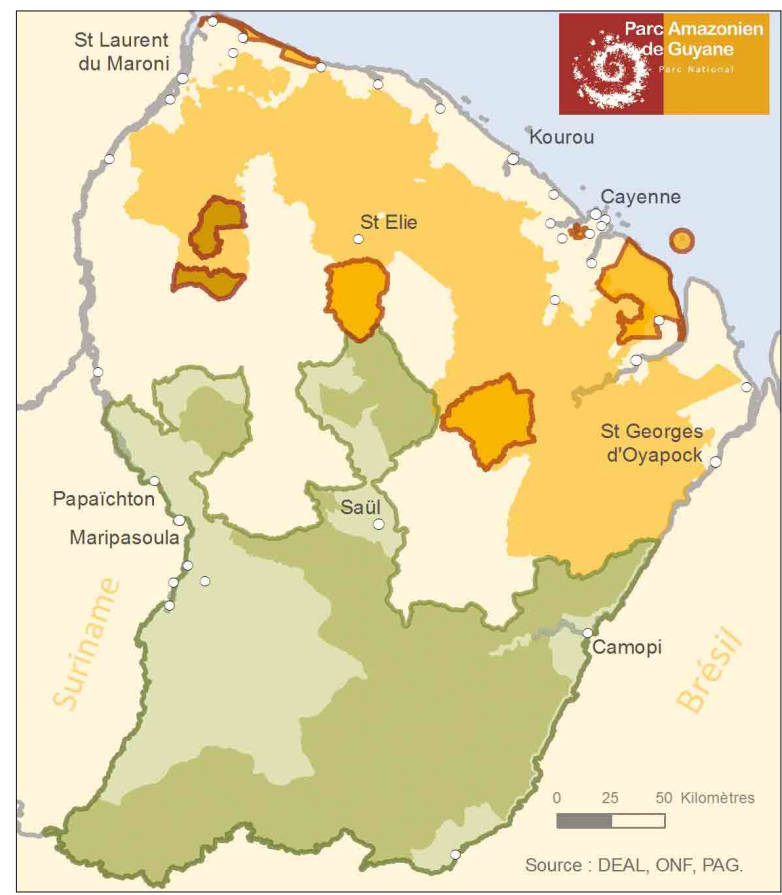

\section{FIGURE 3 \\ CARTE DE LA GUYANE \\ AVEC LES ESPACES PROTÉGÉS ET LES RÉSERVES BIOLOGIQUES () PAG}

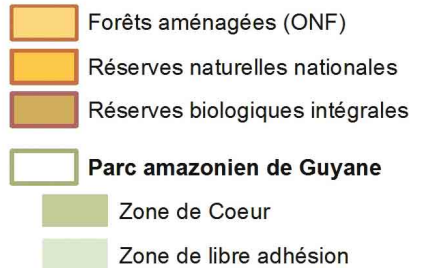

Le parc amazonien de Guyane a pour mission de préserver les patrimoines naturels, de valoriser les cultures traditionnelles et d'accompagner les communautés d'habitants dans la définition d'un projet de territoire et de développement local dans le respect de leurs modes de vie et de l'environnement ; ceux-ci tirent encore une bonne part de leurs ressources du fleuve et de la forêt (photo 1, p. 548).

La forêt amazonienne de Guyane abrite plus de 5200 espèces végétales dont 1200 arbres recensés à ce jour, une faune de plus de 185 espèces de mammifères, près de 300 reptiles et batraciens, environ 720 espèces d'oiseaux, 480 poissons d'eau douce et des dizaines de milliers d'espèces d'insectes (photo 2, p. 548). Le parc national possède des milieux remarquables et originaux comme les savanes-roches, les inselbergs (photo 3, p. 548) et les monts forestiers audelà de 500 mètres d'altitude caractérisés par un très fort taux d'endémisme.

Certains groupes taxonomiques sont peu connus : champignons, plantes vasculaires, insectes, batraciens et reptiles. Une espèce d'arbre nouvelle pour la science a été découverte en 2009 sur le territoire du parc national (photo 4, p. 548). 


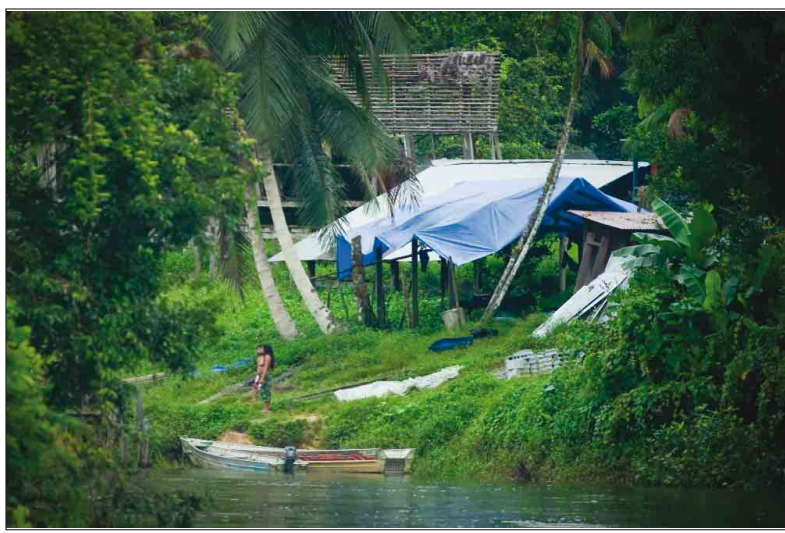

Photo 1 Partie de village amérindien à Trois-Sauts sur le Haut-Oyapock. (c) G. FEUILLET / PAG

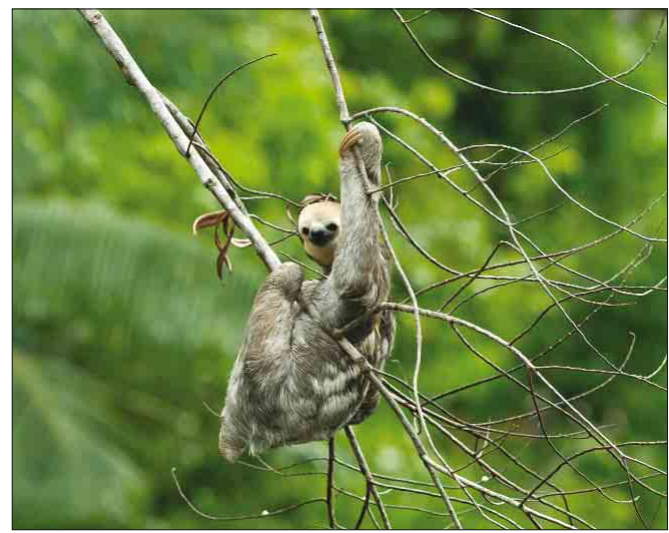

Photo 2 Paresseux à trois griffes (Bradypus tridactylus).

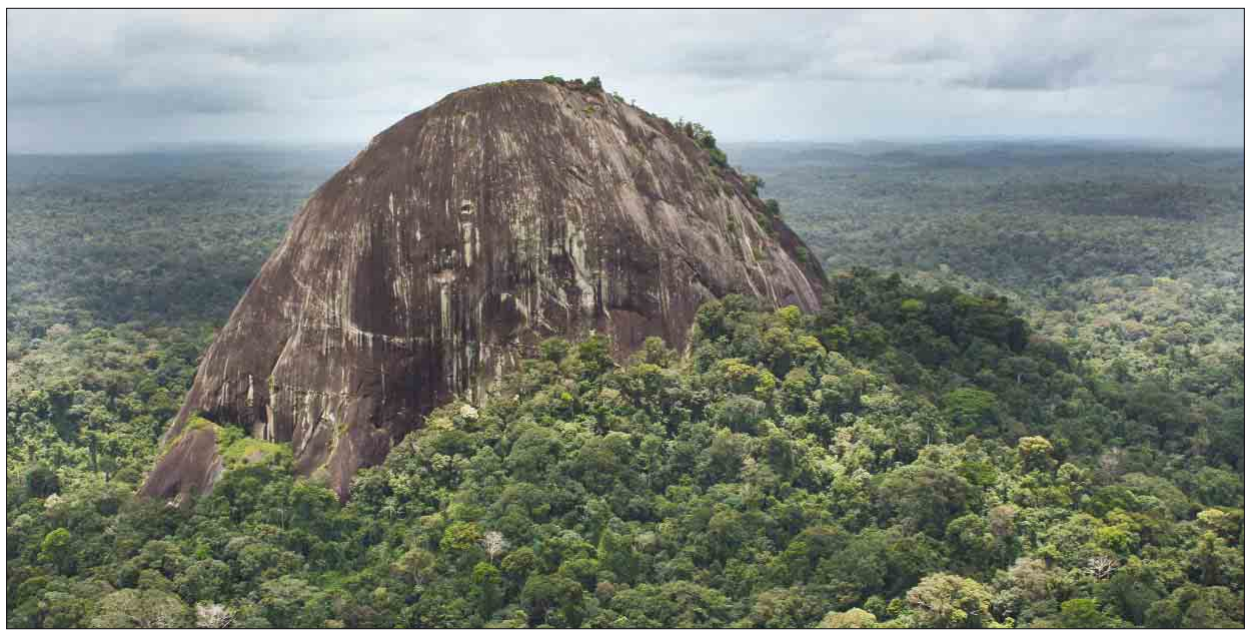

Photo 3 Le territoire du parc amazonien de Guyane abrite des habitats remarquables. Les monts rocheux émergeant de la forêt (inselbergs) connaissent notamment de forts taux d'endémisme. Ici la roche Koutou.

(c) N. SURUGUE / PAG

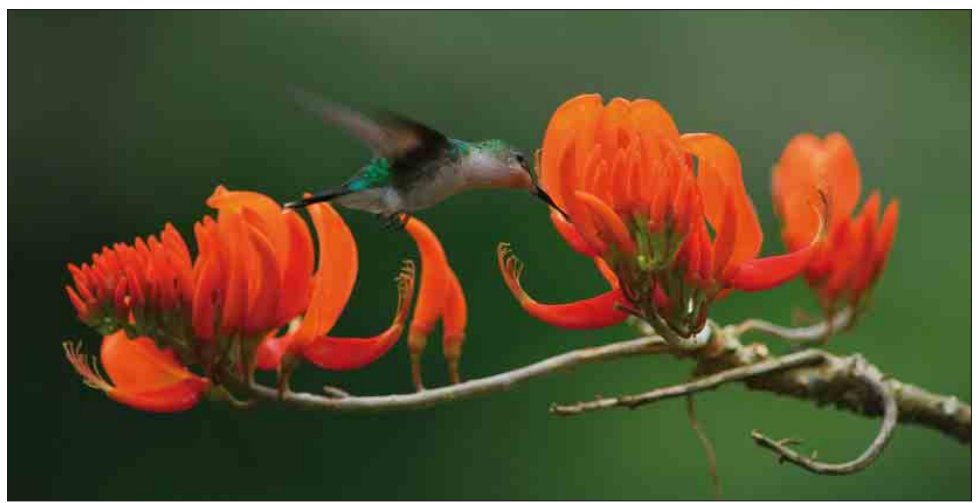

Photo 4 Colibri

(Thalurani furcata)

se régalant du nectar

d'une Erythrine

(Erythrina poeppigiana), arbre rare découvert en 2009 sur le territoire du parc amazonien de Guyane.

(c) T. DEVILLE / ECOBIOS 
La connaissance sur la dynamique et le fonctionnement des écosystèmes est encore fragmentaire. Les habitats naturels forestiers présentent une forte naturalité et un bon état de conservation, ils sont un laboratoire pour la recherche sur les écosystèmes amazoniens. En 2011, le conseil scientifique a validé la première politique de connaissance de l'établissement du parc national.

Les communautés d'habitants (Amérindiens, Bonis...) vivent encore dans une étroite relation avec la nature. À titre d'exemple, les habitants du Sud produisent un artisanat remarquable, de nombreux objets sont fabriqués à des fins d'usage domestique, cultuel ou rituel à partir de ressources naturelles du territoire : arouman, argile, plumes, bois... (photo 5, ci-dessous). La pharmacopée traditionnelle est pratiquée. Les savoirs et savoir-faire constituent un patrimoine unique où la transmission intergénérationnelle est un enjeu capital.

La principale menace qui pèse sur le territoire du parc national est l'orpaillage clandestin : c'està-dire l'exploitation illégale de l'or principalement par les étrangers en situation irrégulière provenant du Brésil. Cette pratique est un désastre écologique tout d'abord, par destruction des cours d'eau, de la continuité hydraulique et du colmatage des cours d'eau par les matières en suspension (photo 6, ci-dessous), du déstockage du mercure natif et de l'apport de mercure par les orpailleurs pour extraire l'or (un peu plus de $1 \mathrm{~kg}$ de mercure pour $1 \mathrm{~kg}$ d'or extrait).
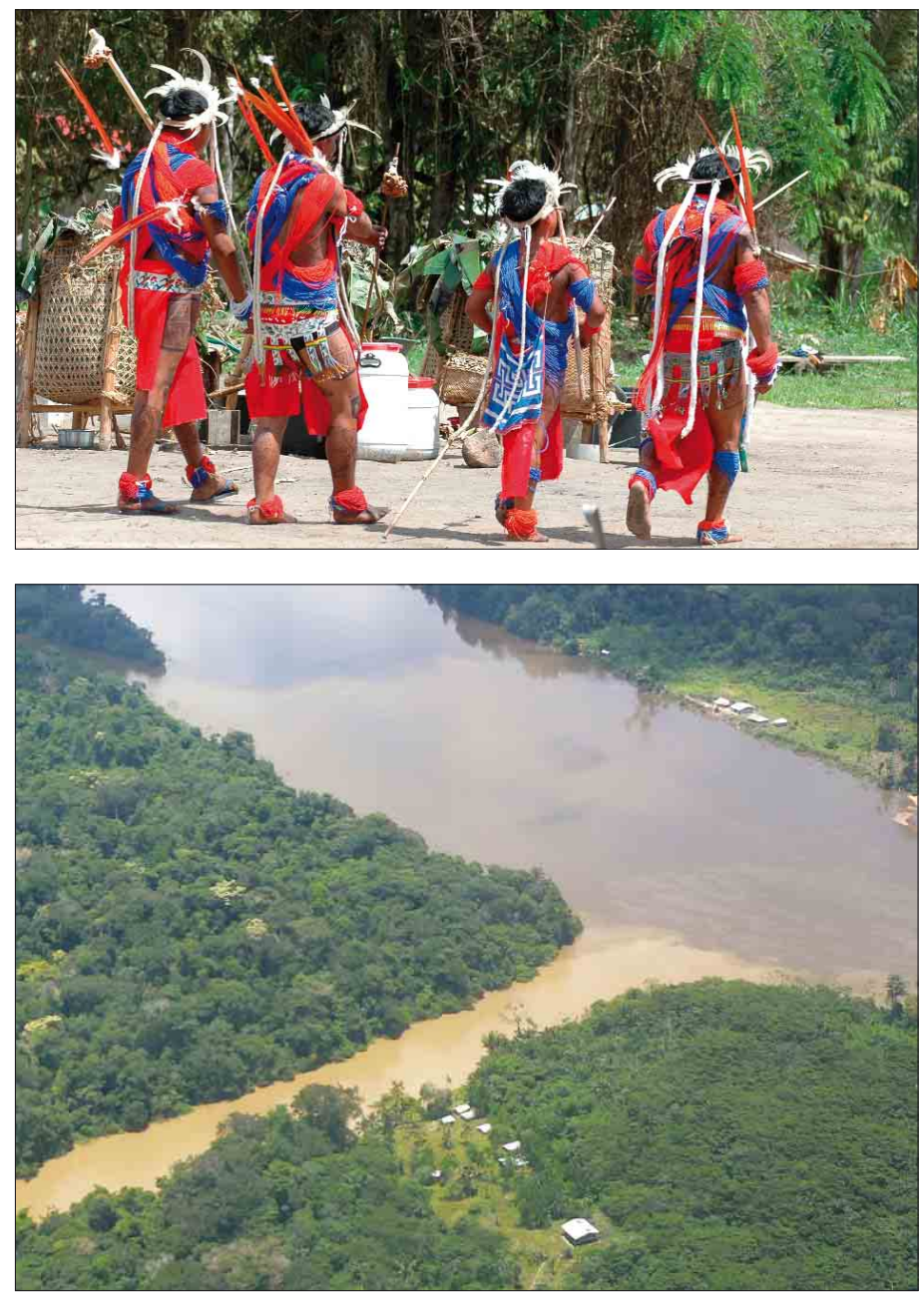

Photo 5 La candidature du rite du maraké wayana-apalaï au patrimoine immatériel de l'UNESCO est soutenue par le parc amazonien de Guyane.

(c) H. GRIFFIT
Photo 6 Les boues rejetées par l'orpaillage illégal alluvionnaire polluent les rivières. Pour les populations vivant à proximité, qui tirent profit de l'eau du fleuve ainsi que de la pêche vivrière, c'est un facteur déstabilisant dramatique. C'est aussi un désastre écologique. Ici, Grand Inini, sur le bassin du Maroni.

(๖) G. FEUILLET 
À cela s'ajoute la pollution par les hydrocarbures et les déchets laissés sur place. C'est aussi un désastre sociétal car l'orpaillage illégal s'accompagne de vols de pirogues et de moteurs, du pillage des abattis, de trafics divers (alcool, armes...), de la prostitution, du braconnage... Le parc national est fortement engagé à travers un programme de diagnostic, de surveillance et d'alerte des autorités (photos 7 et 8 , ci-dessous).

Ici, la protection des populations, la préservation de l'intégrité des milieux naturels et de la biodiversité revêtent un enjeu de souveraineté nationale et de sécurité intérieure (photo 9, cidessous).

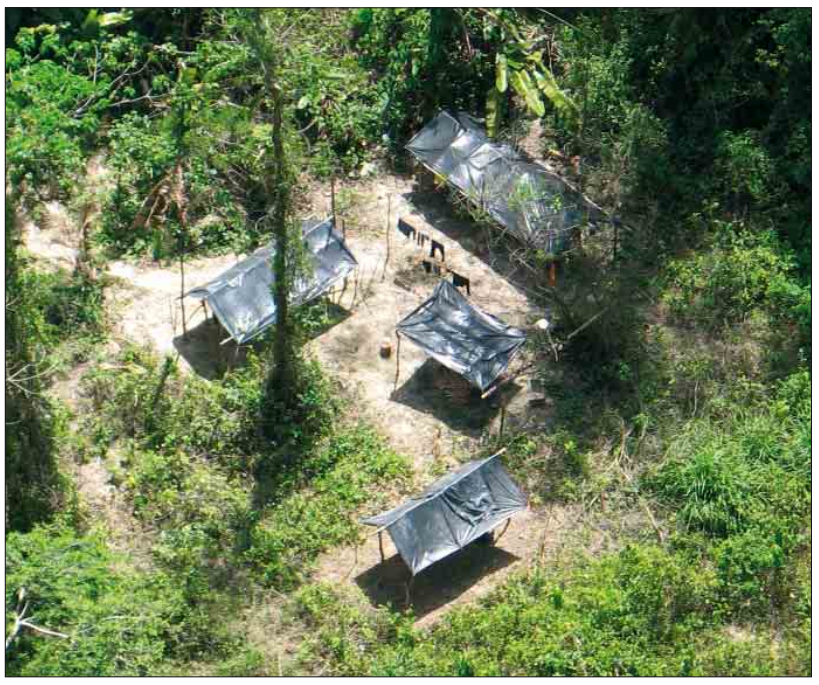

Photo 7 La pression exercée par l'État sur les orpailleurs clandestins les pousse à travailler en petites unités ici identifiés par le Parc amazonien de Guyane à Saül.

(C) PAG / DTC

Photo 8 Matériel abandonné par les orpailleurs clandestins et mis à jour par les agents de Camopi du Parc national.

() PAG / DTO
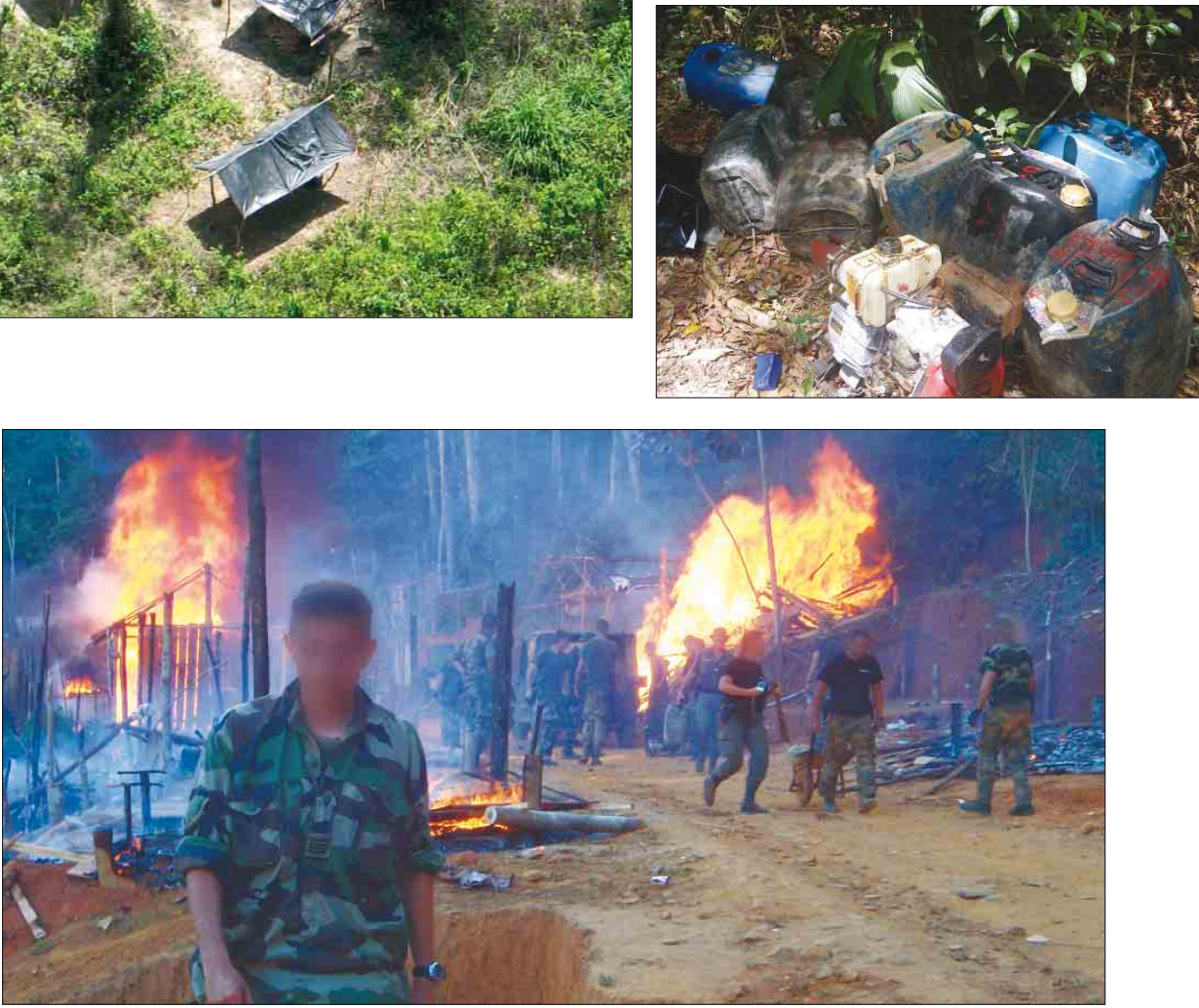

Photo 9 Scène d'apocalypse au cœur de la forêt amazonienne.

Destruction d'un chantier d'orpaillage clandestin par les forces armées de Guyane ( $9^{\mathrm{e}}$ RIMA) et la Gendarmerie nationale. 


\section{3 - Biodiversité et gestion des forêts publiques en Guyane}

L'ONF a sous sa responsabilité en Guyane une surface de 5,6 millions d'hectares située principalement dans la moitié nord du territoire (figure 3, p. 547).

Seule forêt équatoriale de l'Union européenne faisant partie d'un des 15 grands massifs non fragmentés, cette forêt concentre des enjeux de portée internationale.

La gestion de ces espaces vise la préservation :

- de la diversité biologique, en très bon état de conservation, mais faisant l'objet de menaces localisées,

- des stocks de carbone forestier,

- des ressources forestières et leur mise en valeur au profit du développement endogène (80 $000 \mathrm{~m}^{3}$ par an),

- du rôle joué par la forêt pour les communautés autochtones qui en dépendent.

L'ordonnance du 28 juillet 2005 et le décret du 14 novembre 2008 ont complété le dispositif législatif et réglementaire et donné un fondement juridique à la gestion de la forêt. Ils étendent à ce département l'essentiel du Code forestier, adapté aux spécificités locales. Après un premier ensemble de 2,3 millions d'hectares en 2008, l'ONF proposera dans les années à venir les autres forêts, en particulier de la bande littorale, devant relever du Code forestier.

\section{La conservation de la biodiversité commence par la protection du foncier et la lutte contre les occupations illégales...}

La surveillance de l'ONF, en relation étroite avec le parquet, permet de stabiliser et de contenir les défrichements illégaux. Les forêts sont en effet sous pression constante sur la bande du littoral et à proximité des voies de pénétration.

Le contrôle de l'activité minière et la lutte contre l'orpaillage illégal au travers notamment d'un observatoire de l'activité minière (OAM), basé sur le traitement par SIG d'images satellitaires et de missions héliportées de diagnostic sont deux activités prioritaires. Cet observatoire piloté par l'ONF couvre tout le territoire, dont le parc national, et permet le renseignement régulier du préfet et des forces de l'ordre ; il permet aussi de dresser des bilans interannuels des impacts de cette activité illégale.

\section{... et se poursuit par une gestion durable des ressources naturelles...}

Le travail des forestiers guyanais continue d'être un travail de pionnier, avec la définition progressive de règles de gestion et d'exploitation qui relèvent de l'exploitation à faible impact, selon la définition de la FAO «opération d'exploitation forestière intensément planifiée, précautionneusement mise en œuvre et contrôlée afin de minimiser son impact sur le peuplement et les sols forestiers, et se basant habituellement sur une sélection des individus à abattre». Ainsi, la récolte limitée à 4 à 5 tiges/ha représentant 20 à $25 \mathrm{~m}^{3}$ est précédée systématiquement d'un inventaire en virée avec apposition de plaquettes et repérage par GPS et d'une optimisation du réseau de pistes. Ce travail d'optimisation nécessite la poursuite de travaux de recherche, réalisés en partenariat avec les organismes présents en Guyane (IRD, AgroParisTech, CIRAD...).

... sans oublier la gestion et l'étude des aires protégées comme les réserves naturelles ou biologiques (encadré 1 “Les réserves biologiques” p. 546, et photo 3, p. 548). 


\section{Les enjeux à venir}

- Les actions de protection, en particulier contre l'orpaillage illégal, doivent être maintenues et consolidées, avec le soutien indispensable de l'État, malgré toute la difficulté de l'entreprise.

- La certification de la gestion forestière (PEFC et FSC pour partie), attendue pour 2012, devrait, au travers de la charte de bonnes pratiques d'exploitation forestière, être un élément important dans la démarche d'amélioration continue de la qualité de la gestion forestière.

- La coopération internationale et les échanges avec les pays voisins, en particulier le Brésil, concernant la gestion durable des forêts équatoriales et la préservation des stocks de carbone constituent des éléments également nécessaires.

- L'association des collectivités à la gouvernance de ces forêts domaniales est à renforcer ainsi que la rétrocession de certaines d'entre elles aux collectivités locales.

\section{4 - Des 50 pas géométriques à la forêt du littoral}

Moins connue que la forêt humide présente en altitude mais encore beaucoup plus fréquentée, la forêt du littoral est présente de façon disjointe sur l'ensemble des côtes de la Martinique et de l'archipel de Guadeloupe. Ces espaces représentent une surface de forêt domaniale de 1500 ha en Guadeloupe et de 1800 ha en Martinique et constituent avec les terrains du Conservatoire du littoral gérés principalement par l'ONF et le parc national de Guadeloupe, les sites littoraux les plus remarquables des Antilles françaises.

\section{Une histoire, un statut}

Pour des raisons économiques et surtout stratégiques, en 1674 , le roi de France décida de rattacher à son domaine une zone littorale dite "des 50 pas du Roy », désignée sous le nom de « 50 pas géométriques ». Cette zone représente une bande de 81,20 mètres de large à partir du rivage. L'objectif était de rendre plus difficile l'abord des îles par le maintien d'une zone boisée permettant la libre circulation le long du littoral et supportant les batteries de défense des rades et accès.

Après la Révolution, les « 50 pas » devinrent domaine public de l’État. Dans les années 1950, un changement de statut fit passer les « 50 pas » dans le domaine privé de l'État. Les occupations illicites, les extractions abusives de sable et la dégradation des plages se multipliant, ont motivé l'affectation des portions du littoral encore peu dégradées au ministère chargé de l'Agriculture pour les soumettre au régime forestier (en 1979 à la Martinique, en 1975, 1976 et 1980 à la Guadeloupe).

Ce sont ces terrains qui constituent aujourd'hui une bonne part de la forêt domaniale du littoral, domaine privé de l’État. 
À compter de 1996, a été également engagé un transfert progressif et contrôlé d'une partie des terrains appartenant au domaine public maritime au Conservatoire du littoral. Le Conservatoire du littoral confie leur gestion par voie de convention à l'ONF, au parc national de la Guadeloupe pour la gestion écologique et aux communes pour l'animation et la politique d'accueil du public.

Une partie du domaine public maritime et lacustre (mangroves) a été également remis en gestion au Conservatoire du littoral.

\section{Des paysages et un patrimoine naturel remarquable}

Les principales formations rencontrées de la forêt du littoral sont xérophiles, pionnières ou rabougries. Elles abritent une faune variée composée notamment d'oiseaux et de tortues fréquentant les nombreuses plages, sites de pontes. Certaines zones sont particulièrement dégradées, la forêt étant souvent considérée comme un espace appartenant à celui qui l'occupe. Les empiétements par les cultures, les commerces et les constructions sont nombreux. Les gestionnaires s'emploient donc depuis des décennies à lutter contre toutes ces atteintes qui menacent le milieu naturel. Les résultats, incontestables, sont cependant encore fragiles et réversibles.

Les mangroves, formations boisées dominées par les palétuviers en eaux saumâtres, sont aussi originales et fragiles que peu répandues ( 1800 ha à la Martinique, 6200 ha en Guadeloupe). La forte pression anthropique (pollution, développement d'infrastructures) nécessite une protection et une veille constante de la part des gestionnaires.

\section{Les objectifs de gestion}

La gestion de la forêt du littoral est basée sur la recherche permanente d'un équilibre entre protection et valorisation.

Les plans de gestion ou les programmes d'aménagement forestier prévoient généralement :

- le bornage et l'entretien des limites des terrains ;

- la protection de la forêt contre les défrichements, les empiètements, les vols de bois et de sable, les abandons d'épaves et de détritus se fait en liaison étroite avec la préfecture, le parquet et les partenaires (dont l'agence des 50 pas à la Martinique) ;

- l'amélioration de l'accueil du public par une meilleure intégration des équipements et par la mise en place de supports d'informations pédagogiques notamment sur la valeur patrimoniale de ces milieux naturels ;

- le reboisement progressif des parcelles cultivées ou anciennement cultivées ;

- la régularisation foncière des zones qui ont perdu leur caractère naturel.

Compte tenu des objectifs fixés, trois zones distinctes de gestion sont généralement prévues : protection physique et paysagère, accueil du public, et intérêt écologique particulier.

La conservation de la biodiversité de la forêt du littoral passe par la protection de l'intégrité du foncier. 


\section{5 - Gestionnaire d'espaces : les enjeux pour le parc national de la Guadeloupe et pour l'ONF aux Antilles}

\section{Le contexte naturel}

Cinq grands types de milieux naturels forestiers sont présents aux Antilles françaises : la forêt dense humide couvrant la majorité du massif montagneux ; la forêt mésophile semi-décidue ; la forêt sèche xérophile sur le littoral ; les milieux secs à très basse altitude et sur les îles ainsi que les mangroves.

Le « cœur historique » de 1989 du parc national de Guadeloupe (PNG) porte sur 17 ooo ha de forêts des hauteurs du massif de l'île volcanique de la Basse-Terre dont le point culminant est le dôme de la Soufrière à $1467 \mathrm{~m}$. La forêt hygrophile est le milieu le plus structuré avec 5 strates distinctes de végétation et une richesse spécifique très élevée (300 à $600 \mathrm{~m}$ d'altitude). Vient ensuite, la forêt altimontaine rabougrie (550 à $850 \mathrm{~m}$ d'altitude) par les effets conjugués des vents et de l'hygrométrie. Au-dessus de $850 \mathrm{~m}$ se développent les hauts fourrés d'altitude dominés par le Mangle montagne et les savanes d'altitude composées de formations difficilement pénétrables où s'entremêlent ligneux, herbacées et épiphytes adaptées à la saturation en eau qui accompagne la très forte couverture nuageuse (jusqu'à 12 mètres de précipitations par an).

La flore du cœur forestier est particulièrement riche et diversifiée : 811 plantes à fleur et 274 fougères, soit plus de $50 \%$ des angiospermes et plus de $90 \%$ des fougères de la flore indigène de la Guadeloupe (Fournet, 2006 in Rousteau, 2007). La forêt abrite quelques espèces

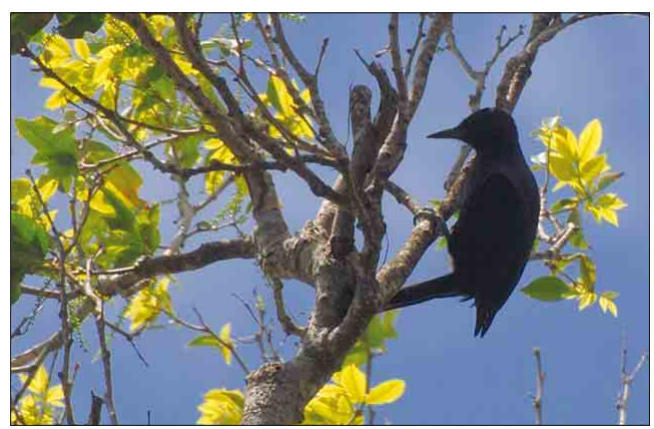
de la faune endémique de la Guadeloupe ou des Petites Antilles telles que le Pic de Guadeloupe, Melanerpes herminieri (photo 10). Trois espèces de chauves-souris sur les 13 espèces présentes en Guadeloupe sont strictement inféo-

Photo 10 Le Pic de Guadeloupe, Melanerpes herminieri, est endémique de cette île et le seul représentant de son groupe pour toutes les petites Antilles.

(c) PARC NATIONAL DE GUADELOUPE

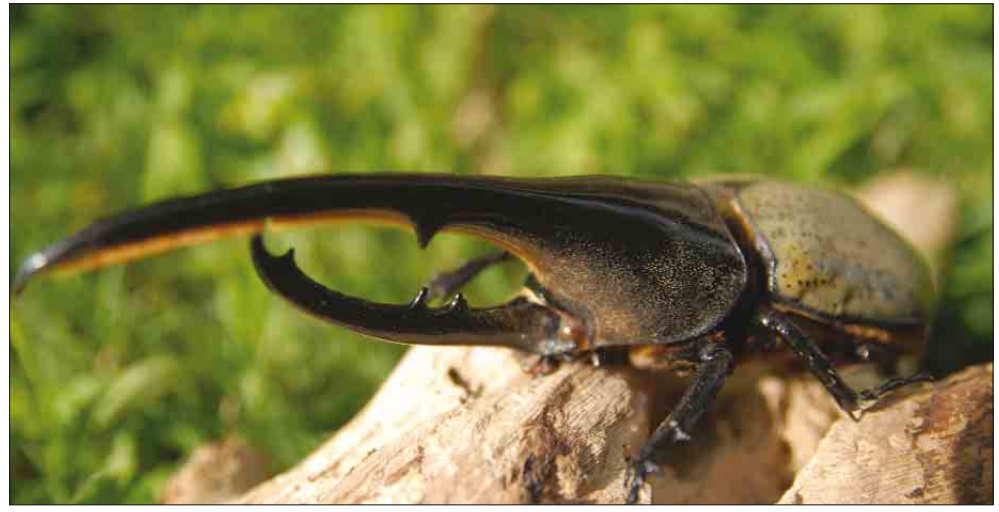

Photo 11 Le Dynaste Hercule, espèce protégée en Guadeloupe car très convoité par les collectionneurs (c) PARC NATIONAL DE GUADELOUPE 
dées aux forêts. Les insectes recèlent une biodiversité endémique originale : par exemple le spectaculaire Dynaste (photo 11, page 554), protégé en Guadeloupe, la Mygale de la Soufrière, ou encore deux petites libellules des torrents d'altitude.

La mise en place en partenariat avec l'ONF, l'université Antilles-Guyane et le CIRAD, d'un réseau de placettes permanentes qui vise à mieux connaître la dynamique spatio-temporelle de la forêt dense humide insulaire fait de ces espaces un terrain d'étude pour toute la Caraïbe.

\section{L'action des gestionnaires}

L'ONF gère aux Antilles 52600 ha soit près de $20 \%$ de la surface de ces départements.

La gestion est prioritairement axée sur les thèmes suivants :

- La préservation du foncier est une action fondamentale pour maintenir l'intégrité des espaces naturels. Elle nécessite un investissement continu (un tiers du temps des agents de l'ONF) majoritairement concentré sur la zone du littoral (plus de 600 occupations, principalement illégales, sont recensées par l'ONF en Martinique).

- La constitution d'un réseau de réserves naturelles et de réserves biologiques complémentaires de l'espace classé cœur de parc afin de gérer, de façon spécifique, les habitats naturels les plus remarquables et les plus représentatifs des Antilles françaises. Par ailleurs, le parc national de la Guadeloupe est chef de file d'un programme de coopération de la zone Caraïbes pour la restauration du Lamantin, et l'ONF d'un programme INTERREG consacré à la mangrove.

- Le développement endogène porte sur deux thématiques principales, le tourisme et la microfilière bois :

- Le tourisme. Compte tenu de la situation et de la qualité paysagère des milieux naturels, ces espaces sont un atout majeur pour l'accueil du public. Il s'agit bien sûr des sites de l'intérieur comme la forêt humide de Basse-Terre en Guadeloupe ou des Pitons du Carbet et de la montagne Pelée en Martinique, mais aussi et surtout les sites littoraux (420 kilomètres de linéaire géré par l'ONF aux Antilles). Les projets se réalisent en association étroite avec les communes, le département, la région, l'ONF et le PNG sur son territoire. Le développement de la randonnée impose un effort d'entretien plus important des sentiers qui parcourent le massif.

- La microfilière bois aux Antilles est fondée sur le Mahogany (Acajou du Honduras), essence introduite de grande valeur technologique, menacée dans son milieu d'origine et inscrite à l'annexe II de la CITES.

\section{Les enjeux d'avenir}

Le rapport des populations et des usagers à la forêt a changé. Les Guadeloupéens et les Martiniquais s'approprient mieux ce territoire et lui reconnaissent une réelle valeur patrimoniale. Les services écosystémiques rendus par la forêt doivent être mieux étudiés, davantage, et de nouvelles sources de financement sont à trouver.

La gouvernance des espaces forestiers doit renforcer les partenariats et optimiser la recherche et la mutualisation des moyens.

La volonté de faire émerger une filière énergie à partir de la biomasse doit prendre en compte la nécessaire conservation de la biodiversité ainsi que la protection des sols.

Les espaces naturels ont un rôle déterminant à jouer dans les politiques de tourisme qui doivent être respectueuses de l'environnement et des populations. 


\section{6 - Le parc national de la Réunion et l'Office national des forêts : conservation de la biodiversité et développement endogène}

Le cœur du parc national de la Réunion regroupe $94 \%$ des habitats primaires et représente $42 \%$ de la superficie de l'île (105 447 ha). Ce vaste ensemble d'un seul tenant présente plusieurs gradients dont un gradient altitudinal qui va de l'océan jusqu'au sommet du piton des Neiges à $3071 \mathrm{~m}$, plus haut sommet des Mascareignes. Plus de 43000 ha sont encore constitués d'espaces où l'emprise humaine reste peu visible, distants de plus de $500 \mathrm{~m}$ de tout axe de pénétration, même pédestre (photo 12, ci-dessous).

Ces espaces naturels présentent un taux d'endémisme élevé (34 \% des espèces végétales) et un bon état général de conservation, à l'exception des forêts sèches (photo 13, ci-dessous).

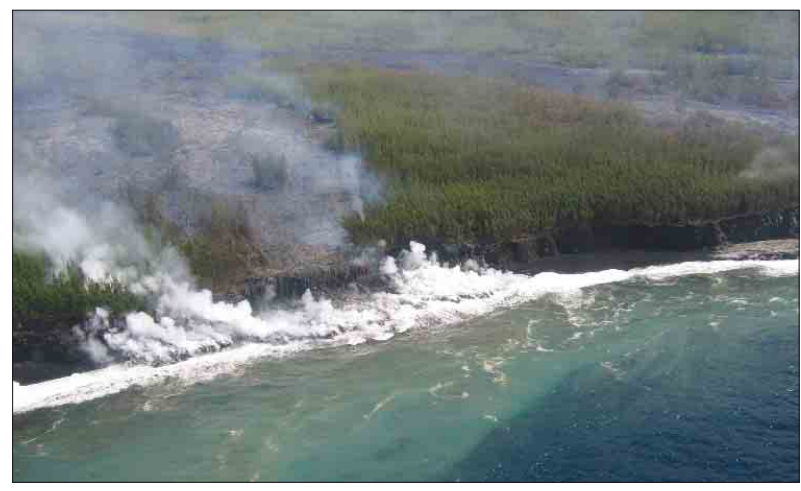

Photo 13 Les îles océaniques présentent un fort taux d'endémisme. Ici, un tectec (Saxicole tectes) en lisière forestière à la Réunion.

() J. TROUVILLIEZ
Photo 12 Les éruptions du piton de la Fournaise (Réunion) constituent des occasions uniques pour étudier les successions végétales.

() J. TROUVILLIEZ

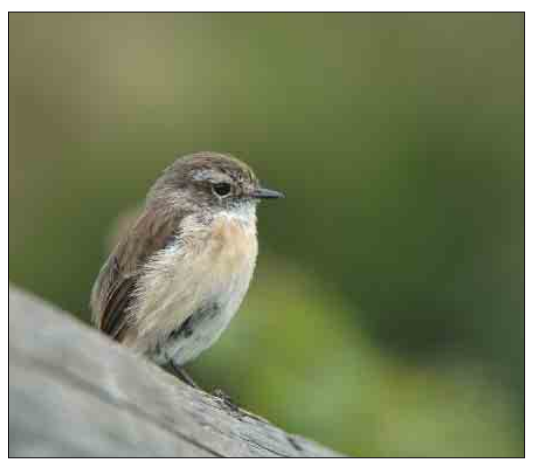

Dans le cœur, coexistent des espaces à vocation principalement naturelle (100 883 ha) ainsi que les îlets habités de Mafate et des Salazes ( 3127 ha) et quelques enclaves cultivées ou accueillant une activité pastorale (1 441 ha). L'attractivité de ces lieux pour des activités de tourisme et loisirs est croissante.

Ces espaces ont été reconnus depuis le $1^{\text {er }}$ août 2010 par l'UNESCO, à la fois du point de vue de la biodiversité et des paysages, par l'inscription de ses " pitons, cirques et remparts » sur la liste des sites naturels du patrimoine mondial.

Ces caractéristiques orientent les actions des forestiers dont les piliers sont la protection et le développement :

- Protection dynamique : la principale menace est l'envahissement des écosystèmes par les nombreuses espèces exotiques (photo 14, p. 557). Ceci exige une action constante, orientée vers la détection et la lutte précoce dans le cadre d'une stratégie générale définie avec l'État, l'ONF, le parc national et le conservatoire botanique national des Mascareignes ; mais aussi vers des travaux plus lourds de lutte et de reconquête utilisant les techniques de génie écologique. Par ailleurs, l'ONF poursuit la mise en place et la gestion d'aires protégées représentatives des différents milieux : sept réserves biologiques domaniales couvrant 13000 ha sont en place. 
- Développement endogène : le développement touristique de l'île constitue un objectif majeur, les espaces naturels forestiers y jouent un rôle primordial. L'ONF est un opérateur important en matière d'équipement et d'accueil pour le public, le tout dans le respect des sites à forte valeur patrimoniale. Ces espaces sont aussi des lieux de solidarité puisque l'ONF y conduit les travaux dans le cadre de chantiers d'insertion financés par le conseil général (500 personnes en contrat d'insertion).

La charte du parc national en cours d'élaboration constituera le projet partagé de territoire et vaudra également plan de gestion du bien inscrit au patrimoine mondial. Elle repose sur une concertation élargie avec de nombreux acteurs publics et privés, dans un souci de mise en cohérence de toutes les stratégies de planification et programmations, notamment en matière forestière.

Au-delà du volet réglementaire qui s'applique au cœur, la charte vise surtout une mobilisation volontaire pour des enjeux et des objectifs prioritaires et partagés tant pour la conservation que pour le développement endogène, autour d'actions concrètes qui ne peuvent aboutir sans un engagement collectif.

Quant à l'ONF, gestionnaire des forêts départementalo-domaniales, une évolution profonde de la gouvernance a vu le jour ces dernières années avec la mise en place d'une concertation très étroite avec le conseil général et un travail de proximité avec les élus.

Les enjeux des années à venir sont :

- Parfaire le partenariat entre le parc national et l'ONF.

- Pour le parc national, protéger et évaluer l'action en faveur des espèces et des habitats naturels menacés d'une part et renforcer la pédagogie et la communication au profit de la biodiversité d'autre part. Enfin, renforcer l'appui, l'ingénierie et la fonction d'ensemblier de l'établissement public auprès des collectivités en particulier dans le domaine du développement local.

- Pour l'ONF, la forêt, lieu d'insertion sociale, doit également devenir le support d'emplois pérennes plus nombreux, en accompagnant le développement du tourisme, ainsi qu'en créant de nouveaux services dans les espaces les moins sensibles pour la protection de la nature (ecolodges, camping nature, bike park.). Il est prévu le maintien d'une production de bois d'œuvre pour assurer la pérennité d'une microfilière d'ébénisterie (Tamarin) et de charpente et bois de coffrage (Cryptoméria), mais également de développer une filière biomasse, dans le respect de la biodiversité, afin de contribuer à l'objectif d'autonomie énergétique affiché pour l'île de la Réunion à l'échéance de 2030.

- Enfin, la protection contre les risques (incendie et autres risques naturels) reste également une composante majeure, ainsi que cela a été illustré par l'incendie d'octobre 2011. Cette protection fait actuellement l'objet d'une réflexion afin d'améliorer l'efficacité de la lutte et la coordination de l'ensemble des acteurs concernés.

Photo 14 Les forêts de la Réunion sont progressivement envahies par un cortège d'espèces exotiques envahissantes, ici au premier plan le Longose.

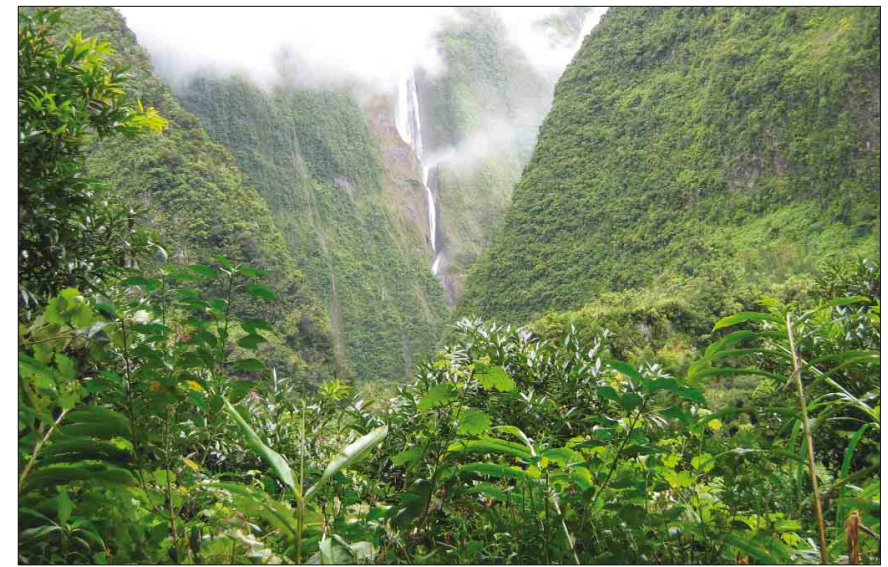




\section{BIBLIOGRAPHIE}

CADET (T.). - La Végétation de l'île de la Réunion. Étude phytoécologique et phytosociologique. - Université d’Aix-Marseille III, 1977. (Saint-Denis-de-la-Réunion : Imprimerie Cazal, 1980).

CHARLES DOMINIQUE (P.). - Guyane Milieux, faune, flore. - CNRS-PAG-PNRG-DIREN, CNRS Édition, 2011. - 219 p.

COMITÉ FRANÇAIS DE L'UICN, OFFICE NATIONAL DE LA CHASSE ET DE LA FAUNE SAUVAGE. - Les vertébrés terrestres introduits en outre-mer et leurs impacts. - Comité français de l'UICN ; Office national de la chasse et de la faune sauvage, 2011. - 100 p.

DIAMOND (J.M.). - Collapse : How Societies Choose to Fail or Succeed. - Viking ed., 2005. - 592 p.

MACARTHUR (R.H.), WILSON (E.O.). - The theory of island biogeography. - Princeton (USA) : Princeton University Press, 1967.

MYERS (N.), MITTERMEIER (R.A.), MITTERMEIER (C.), FONSECA (G.A.B. da), KENT (J.). - Biodiversity hotspots for conservation priorities. - Nature, 403, 2000, pp. 853-858.

PONTEAU (R.), MEYER (J.-Y.), TAPUTUARAI (R.), STROLL (B.). - La Fonte de la biodiversité dans les îles : modélisation du réchauffement global sur la végétation orophile de Tahiti (Polynésie française). VertigO, 10 (3), décembre 2010, pp. 1-15.

ROLLET (B.). - Arbres des Petites Antilles. - Office national des forêts, 2010. - 2 tomes : 276 p. + 914 p. ROUSSEL (E.). - Les mangroves de l'outre-mer français. - Rapport Conservatoire du littoral, 2009. - 142 p.

SOUBEYRAN (Y.). - Espèces exotiques envahissantes dans les collectivités françaises d'outre-mer. État des lieux et recommandations. - Paris : Comité français de l'UICN, 2008. - 204 p. (Collection Planète nature).

TSAYEM DEMAZE (M.T.). - Croissance démographique, pression foncière et insertion territoriale par les abattis en Guyane française. - Norois, 206 (1), 2008, pp. 111-127.

WILLIAMSON (M.). - Biological invasions. - Chapman et Hall, 1997.

\section{LES FORÊTS EN OUTRE-MER : UN ENJEU MONDIRL POUR LA BIODIUERSITE [Résumé]}

Les forêts en outre-mer sont très variées et abritent une grande richesse biologique, souvent unique, car les taux d'endémicité y sont élevés. Quatre des cinq points chauds de la biodiversité française se situent outremer, ce qui illustre la responsabilité mondiale de la France. Ces milieux ont vu au cours du temps leur surface diminuer et leur état de conservation s'altérer par endroits. Les pressions sont diverses : urbanisation, mise en valeur agricole ou orpaillage... La valeur de ces forêts ultramarines est mieux reconnue désormais et de nombreuses actions sont menées dans les territoires : création d'aires protégées, lutte contre les espèces envahissantes, meilleure valorisation au travers de la filière bois ou de l'écotourisme mais les menaces restent vives et la sécurisation du foncier apparaît comme une priorité pour mener à bien les politiques publiques de conservation. La connaissance du fonctionnement des écosystèmes, encore fragmentaire, est une nécessité pour accroître l'efficacité des actions de gestion et de conservation entreprises.

Enfin, la mutualisation et la coordination des efforts des acteurs publics dans le cadre d'une approche globale et en réseau de la conservation seront déterminantes pour l'avenir.

\section{FORESTS IN OUERSERS TERAITORIES - GLOBAL IMPLICATIONS FOR BIODIUERSITY [Abstract]}

The forests in overseas territories are highly varied and shelter huge and often unique biological riches due to high rates of endemicity. Four of the five biodiversity hot spots in France are located in overseas territories, making for the country's global responsibilities in this area. Over time, their surface area has decreased and their conservation status has changed in some places. They are under various forms of pressure: urbanisation, agricultural development, gold digging, etc. The value of these overseas forests is now better recognized and many measures have been undertaken in these territories: creation of protected areas, control of invasive species, enhanced productivity through forest-based industry uses and ecotourism. Nonetheless, they are still seriously threatened and secure land tenure system appears to be a pre-requisite for conducting public policies of conservation. Better knowledge of how the ecosystems function, which is still very fragmentary, is essential to enhance efficiency of the management and conservation measures undertaken.

Finally, it is crucial for the future that the efforts of public players be pooled and coordinated within the framework of an overall, network-based approach to conservation. 